\title{
* Nursing Education amidst COVID-19: Obstacles and Way Forward
}

\author{
Farida Bibi Mughal1, Nasreen Rafiq ${ }^{1}$, Bibi Hajira Irshad Ali²
}

\author{
${ }^{1}$ Aga Khan University School \\ of Nursing and Midwifery \\ 2 Shifa Tameer-e-millat \\ University, College of Nursing \\ Corresponding Author: \\ Farida Bibi Mughal \\ Email: farida.bibi@aku.edu
}

$\mathrm{T}$ he spread of COVID-19 has infected a lot of people in the world and have changed the dynamics globally in every sphere of life. It has shown devastating effects on educational institutes especially to the nursing education. Future nurses' education and training is of utmost significance as they are the backbone of the healthcare system. In this pandemic, nursing students' education needs an important consideration. This paper intends to review the obstacles faced by nursing institutes to carryout teaching theoretical component along with the clinical practice in nursing education during the COVID-19 pandemic. Also, it recommends ways to continue disseminating quality education among nursing students.

The people around the world have been adapting in every domain of their life with the advent of COVID19. The change can be considered as a positive sign in life but with the current circumstances especially with reference to education, this change is challenging for almost every teaching institute. The challenges begun when all the educational institutes were shut down, later, on re-opening, the number of positive cases among students and staff created an additional stress and online teaching- an evolving and only option of teaching, became a greatest challenge for students as well as teachers (1).

Around the globe, especially in the developing countries, traditional mode of education that is blackboard teaching or classroom teaching was considered as the best way of learning (2). This pandemic has led us to re-think about the other possible ways of teaching to continue the process of educating our youth.

Nursing education along with the other disciplines of healthcare are struggling with the mode of education to date. ${ }^{1}$ Today, when every educational organization is moving towards e-learning, nursing education is trying to find avenues for better quality of education. Elearning can be defined as learning from electronic medium commonly through internet (1). E-learning has become answer to the only question that how students will be getting education in this pandemic? However, in nursing education it cannot be considered as the sole mode of teaching method (3).

Like other disciplines of healthcare, nursing education curriculum includes theoretical knowledge along with clinical practice. The nursing students today will become the future registered nurses in the healthcare sector tomorrow. Therefore, they need to be equipped with the knowledge as well as skills to be able to perform better as professional nurses. Nursing is one of the disciplines which includes human care-giving activities (3). The theoretical component in nursing education can be shifted to e-learning mode and mostly organizations have been practicing it where students learn by attending online classes, listening to the recorded lectures, and availing online resource materials (1). Whereas, in clinical area, nursing students require the skills which are necessary to look after their patients in hospital setting. Manual dexterity comes with more and more practice (4). Hence, the concepts 
can be taught and learned through online medium, but skills cannot be learned in the sameway.

Also, while dealing with the real patients during nursing education, students get a chance to learn and understand human emotions. They can be empathetic and able to connect with the patients when they visualize their sufferings. Furthermore, they get a chance to learn the routine activities and tasks required to be performed in each shift. Such variety of experience is not possible if a student learns through online medium. Therefore, application of theoretical knowledge into practice is questionable nowadays.

In the developing countries, online learning brings a huge challenge of internet connectivity (5). Due to low internet bandwidth students might find it difficult to stay connected for the classes and might miss the important discussion most of the time. This raises question about students' learning in the current situation. Moreover, at the end of each year in nursing education promoting a student to the next year is another emerging concern during pandemic. Nowadays creating an authentic assessment which can evaluate students' learning outcomes is becoming a significant concern among educators (5). With that, academic integrity comes into picture when we think about online assessments in this pandemic time. Along with the aforementioned obstacles currently faced by nursing education system, training of teachers to conduct online classes and assessments also needs important consideration. If the teachers won't be trained enough and if they won't be provided with appropriate tools and resources, then they won't be able to deliver the quality content to the students.

The current educational situation during this pandemic possesses various implications for future. The nursing education system should explore different simulationbased learning software for clinical education to assist and engage the nursing students in online clinical learning and virtual practice of their clinical skills before entering the real hospital environment. Moreover, the use of these digital learning software should be made a part of regular nursing curriculum to give students an exposure of virtual patients and associated nursing management prior to their interaction with real patients. It should also be ensured that the teachers are trained to deal with the digital tools before introducing the variations in order to ascertain the smooth simulation of clinical objectives and improve learning outcomes. Besides, the nursing schools should also introduce a hybrid model approach as their teaching strategy. This approach combines the digital teaching with the traditional face-to-face teaching into a coherent student experience (3). Virtual field visits to different hospital would serve as another learning experience as it would acquaint the students to learn management at different set-ups. Further, the students should be taken to observe various telemedicine clinics at their own settings. It will provide them with the opportunities to see a variety of patients through virtual platforms. Also, all these online education approaches should be made a part of the institutes' learning management systems (MOODLE) to synchronize the strategies with the regular teaching learning modalities to enhancelearning and strengthen student - patient interactions.

In conclusion, the pandemic has brought dynamics in education system and raised various learning opportunities for the education system in the country. It has also given nursing institutes the responsibility to carefully craft their modalities and introduce several innovations in education to improve their students' theoretical knowledge and clinical skills in order to uphold the standard of nursing education globally.

Funding: There was no funding available for this paper.

Conflict of interest: We declare that there were no competing interests.

\section{References}

1. Kumar BS, Wotto M, Belanger P. E-learning, M-learning and D-learning: Conceptual definition and comparative analysis. E-Learning and Digital Media. 2018 Jul;15(4):191-216.

2. Chang CC, Shu KM, Liang C, Tseng JS, Hsu YS. Is blended e-learning as measured by an achievement test and self-assessment better than traditional classroom learning for vocational high school students?. International Review of Research in Open and Distributed Learning. 2014;15(2):213-31.

3. Presti CR. The flipped learning approach in nursing education: A literature review. Journal of Nursing Education. 2016 Apr 28;55(5):252-7.

4. Kuzgun H, Denat Y. The manual dexterity of nursing students and factors that affect it. International Journal of Occupational Safety and Ergonomics. 2018 Apr 5.

5. Smith GG, Passmore D, Faught T. The challenges of online nursing education. The Internet and Higher Education. 2009 Jun 1;12(2):98-103. 